\title{
Real-time ligation chain reaction for DNA quantification and identification on the FO-SPR
}

\author{
Karel Knez ${ }^{1}$, Dragana Spasic ${ }^{1}$, Filip Delport, Jeroen Lammertyn* \\ BIOSYST-MeBioS, KU Leuven - University of Leuven, Willem de Croylaan 42, B-3001 Leuven, Belgium
}

\section{A R T I C L E I N F O}

\section{Article history:}

Received 6 June 2014

Received in revised form

26 August 2014

Accepted 27 August 2014

Keywords:

Ligation chain reaction (LCR)

Fiber Optic Surface Plasmon Resonance (FO-

SPR)

FO-SPR melting assay

Gold nanoparticles (Au NPs)

\begin{abstract}
A B S T R A C T
Different assays have been developed in the past years to meet point-of-care diagnostic tests requirements for fast and sensitive quantification and identification of targets. In this paper, we developed the ligation chain reaction (LCR) assay on the Fiber Optic Surface Plasmon Resonance (FOSPR) platform, which enabled simultaneous quantification and cycle-to-cycle identification of DNA during amplification. The newly developed assay incorporated FO-SPR DNA melting assay, previously developed by our group. This required establishment of several assay parameters, including buffer ionic strength and thermal ramping speed as these parameters both influence the ligation enzyme performance and the hybridization yield of the gold nanoparticles (Au NPs) on the FO-SPR sensor. Quantification and identification of DNA targets was achieved over a wide concentration range with a calibration curve spanning 7 orders of magnitude and LOD of 13.75 fM. Moreover, the FO-SPR LCR assay could discriminate single nucleotide polymorphism (SNPs) without any post reaction analysis, featuring thus all the essential requirements of POC tests.
\end{abstract}

(c) 2014 Elsevier B.V. All rights reserved.

\section{Introduction}

New point-of-care (POC) diagnostic tests are expected nowadays to deliver not only fast and sensitive detection of targets (Gervais et al., 2011) but also target quantification and specific identification of particular mutations. Therefore, many different assays, including quantitative PCR (qPCR) (Cheng et al., 2006) and ligation assays (Sando et al., 2004), have been developed to meet these requirements. Although qPCR has shown extensive utility, as it combines extreme sensitivity with real-time detection and multiplexing capacity (Espy et al., 2006), several characteristics of this assay, among which a high cost, complexity of the assay and fluorescence used to monitor the amplification process in realtime, have limited its application towards POC diagnostics. In the last decade, numerous efforts have been made to use nanoparticles (NPs) in this purpose (Storhoff et al., 2004). Au NPs combine colorimetric read-out with high molecular stability and are therefore ideally suited for integration in POC sensors (Jans and Huo, 2012). However, the sensitivity of Au NP assays remained for years

\footnotetext{
* Corresponding author. Tel.: +32 16 321459; fax: +32 16321614 .

E-mail addresses: knez.karel@gmail.com (K. Knez),

Dragana.Spasic@biw.kuleuven.be (D. Spasic),

Filip.Delport@biw.kuleuven.be (F. Delport),

jeroen.lammertyn@biw.kuleuven.be (J. Lammertyn).

${ }^{1}$ Both authors equally contributed to this manuscript.
}

inferior to qPCR, as the enzymatic amplification of DNA during PCR strongly boosts the detection limit.

Recently, it was shown that Au NPs could be combined with DNA amplification through the ligation chain reaction (LCR) (Shen et al., 2012). In the LCR, DNA is amplified exponentially, much in the same way as in the PCR reaction. However, a thermophilic ligation enzyme is used to covalently bind the phosphorylated 5' end of one DNA hybridization probe with the $3^{\prime}$ end of another hybridization probe, which are only ligated when they are hybridized to a perfectly matching target sequence (Fig. 1). In this way, multiple DNA probes can be linked together even when only one target strand is present, as the complex formed between ligated probes and target is denatured at a temperature above its melting temperature $\left(T_{\mathrm{m}}\right)$, liberating target strands for the multiple subsequent ligation cycles. The reaction results in an exponential amplification, as each ligated target will also function as a template for ligation of reverse probes complementary to the original ligation probes. Using this, Shen et al. (2012) linked together DNA probes immobilized on Au NPs only in the presence of the target sequence. As a result, an increasing amount of Au NPs irreversibly aggregated with increasing number of ligation cycles, causing a gradual shift in the absorbance of the Au NP solution due to LSPR adsorption band coupling. The assay could quantify the initial target concentration by determining the number of ligation cycles necessary to generate a colorimetric change in the Au NP solution. This colorimetric assay had a linear range spanning 6 orders of magnitude and a detection limit of $50 \mathrm{aM}$. 


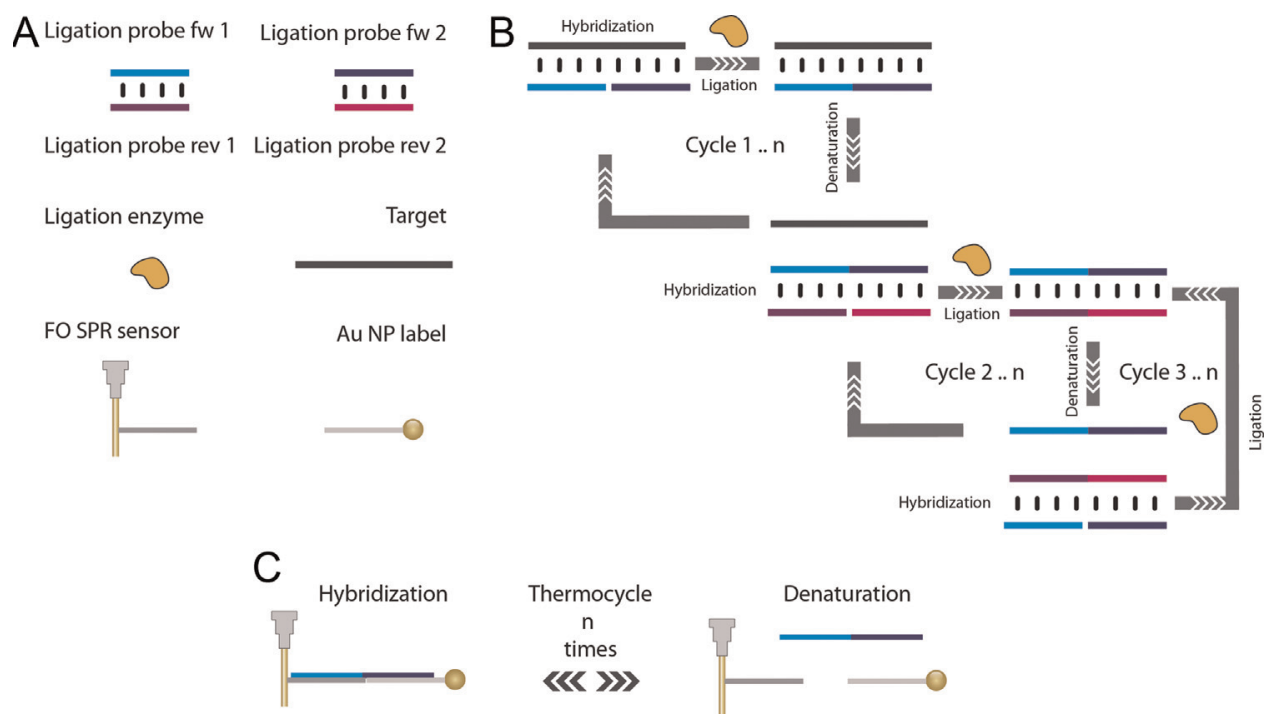

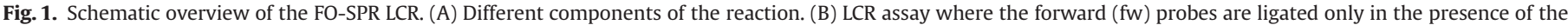

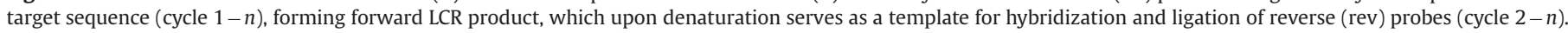

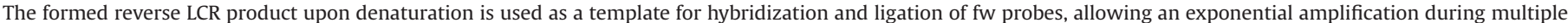

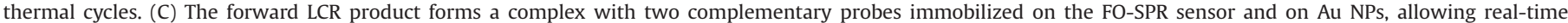
monitoring of the reaction by means of its reaction products.

In this paper, the Au NP LCR is further improved by replacing the colorimetric read-out of the assay, which is limited in measurement speed and sensitivity, with SPR that directly measures Au NP binding and considerably increases assay sensitivity. In this regard, the original assay was redesigned by ligating probes in free solution instead of on Au NPs, which were then binding to complementary probes immobilized on both the Au NP surface and the FO-SPR sensor surface (Fig. 1). Each cycle of the LCR resulted in more ligated probes, allowing more Au NPs to bind to the FO-SPR, which gradually increased the FO-SPR signal from cycle to cycle. LCR products were quantified by counting the number of cycles necessary to reach the amplification threshold. Moreover, the LCR is integrated with the FO-SPR melting assay previously developed in our group (Knez et al., 2012, 2013). By melting the Au NP-ligated probe complex formed on the FO-SPR surface during each LCR cycle, identification of the amplified strands during the LCR becomes possible. SPR technology has been previously implemented for real-time monitoring of DNA melting and hence detection of SNPs (Fiche et al., 2008; Pingel et al., 2012). Although these SPRI chips provide ultrasensitive detection of mutated DNA, melting transitions are not as narrow as those observed on the NP surfaces because the assay monitors the melting behavior of unlabeled DNA strands. The overall benefit of using Au NPs in DNA hybridization and melting assays has been already extensively addressed in the literature (He et al., 2000; Moon et al., 2010; Lytton-Jean and Mirkin, 2005; Oh and Lee, 2011; Knez et al., 2012). Therefore, narrow melting transitions of Au NPs have been combined with SPR real-time monitoring of the DNA melting processes on our in-house developed FO-SPR platform, which is more easily combined with NP solutions compared to microfluidic based SPR layouts. Furthermore, in this approach, Au NPs were used to their full potential as labels in melting analysis and identification of the amplified targets (Jin et al., 2003), contrary to their utilization in the colorimetric LCR Au NP assay as this assay is based on irreversible aggregation of NPs.

The ligation reaction improved the detection limit of the FOSPR melting assay drastically while maintaining its key feature, namely sequence identification and single nucleotide polymorphism (SNP) detection. This represents a significant step towards development of a FO-SPR POC biosensor with capacity to provide sensitive detection of targets, target quantification and specific identification of particular mutations. Furthermore, all the additional features of this in-house developed FO-SPR platform, such as: (1) simplicity of use, as it resembles a concept of one of the most straightforward diagnostic tools available to date, namely 'dipstick' test, (2) possibility for the real-time monitoring of the assay flow with a high measurement sensitivity and high precision temperature control not easily attainable in microfluidic SPR setups and (3) possibility for implementing both DNA- and protein-based bioassays, make FO-SPR platform very attractive technology for development into a diagnostic tool, even applicable in resource limited environments (Knez et al., 2012, 2013; Tran et al., 2013; Pollet et al., 2011).

\section{Materials and methods}

\subsection{Materials}

The chemically synthesized oligonucleotides were purchased from integrated DNA technologies (IDT, Haasrode, Belgium). An overview can be found in Supplementary information (Table S1). In a proof-of-concept experiment, a short 30 bp target was chosen, further referred to as wild type (WT) target, which is a reverse complement of a sequence adopted from the literature (Cao et al., 2005) without real-life functionality. Both capture probes (probe FO-SPR and probe Au NP, Table S1), used to capture the target sequence, are modified with a 3' C3 or 5' C6 thiol modifier (-SH) for immobilization of the oligonucleotides on the FO-SPR sensor and the Au NPs gold surfaces, respectively. They are equipped with either a poly A or poly $\mathrm{T}$ spacer in order to improve the hybridization efficiency of the DNA (Hurst et al., 2006). Citrate stabilized Au NPs, with a mean diameter of $20 \mathrm{~nm}$, were purchased from BBI international (Cardiff, United Kingdom). All chemicals were purchased from Sigma-Aldrich (Bornem, Belgium) unless stated otherwise.

\subsection{FO-SPR sensor fabrication and Au NP functionalization}

The FO-SPR device and sensors were manufactured as previously described in detail (Pollet et al., 2009; Delport et al., 2012) 
and as briefly explained in Supplementary information. Immobilization of thiol-functionalized DNA on Au NPs was achieved by adding activated and purified DNA to a concentrated NP solution. Here, $1 \mu \mathrm{M}$ of $5^{\prime}$ thiol functionalized DNA oligo was first activated with dithiothreitol (DTT, 0.1 M in PB $0.18 \mathrm{mM}, \mathrm{pH} 8.3$ ) to break up thiol dimers that could inhibit the surface functionalization. Activated DNA was subsequently purified using a sephadex column (GE, Oslo, Norway) to remove any traces of active DTT before it was added to the concentrated nanoparticle solution. A fast salt maturation protocol (Hurst et al., 2006) was used to maximize the DNA loading on the Au NPs. Afterwards, Au NPs were washed three times in phosphate buffer with $0.01 \%$ SDS and stored at $4{ }^{\circ} \mathrm{C}$ prior to use.

\subsection{Gold surface backfilling}

Both the FO-SPR sensor and Au NPs were backfilled, prior to use in the LCR assay. Backfilling is a process used to fill in 'empty spots' in between DNA molecules immobilized on a surface, which is often done using alkane thiols with a PEG moiety (Lee et al., 2006). Here, backfilling is performed in order to make the DNA functionalized gold surfaces more stable at elevated temperatures as well as protein repellant (Janssen et al., 2012), preventing enzyme inhibition during the LCR. Both FO-SPR sensors and Au NPs were incubated for $3 \mathrm{~h}$ with a $50 \mu \mathrm{M}$ alkane thiol PEG (Polypure, Oslo, Norway) dissolved in pure ethanol. Afterwards, the FO-SPR sensor surface and $\mathrm{Au}$ NPs were washed 3 times with a 0.01\% SDS phosphate buffer and stored at $4{ }^{\circ} \mathrm{C}$ in phosphate buffer until further use.

\subsection{Ligation chain reaction}

The LCR was performed to link the $5^{\prime}$ phosphate-functionalized Ligation probe FW 1 to the $3^{\prime}$ end of Ligation probe FW 2 (Table S1). A thermophilic ligation enzyme $\left(9^{\circ} \mathrm{N}^{\mathrm{TM}}\right.$ DNA Ligase, New England Bioscience, Ipswich, USA) was used as it can withstand thermocycling of the reaction solution between different temperatures: the probe hybridization temperature $\left(2 \mathrm{~min}\right.$ at $\left.35^{\circ} \mathrm{C}\right)$, the optimal ligation temperature $\left(2 \mathrm{~min}\right.$ at $42.5^{\circ} \mathrm{C}$ ) and the denaturing temperature of the ligation product $\left(5 \mathrm{~s}\right.$ at $\left.70^{\circ} \mathrm{C}\right)$. The thermal ramping speed had to be lowered compared to $5{ }^{\circ} \mathrm{C} / \mathrm{s}$ standardly

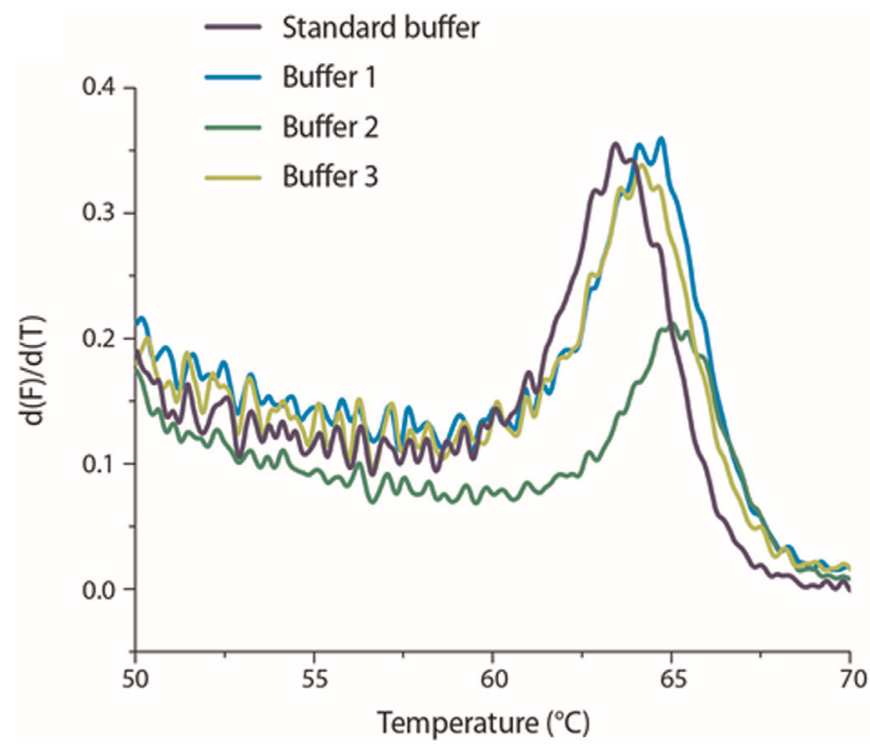

Fig. 2. $q P C R$ HRM following the ligation reaction under different buffering conditions. Whereas buffer 2 drastically lowered the ligation yield, buffers 1 and 3 gave ligation yield comparable to the standard LCR buffer. used in LCR assays to allow a good FO-SPR signal acquisition during DNA melting. Different thermal ramping speeds were tested for achieving reasonable speed and thus safeguarding the total assay time $\left(1{ }^{\circ} \mathrm{C} / \mathrm{s}, 0.5^{\circ} \mathrm{C} / \mathrm{s}\right.$ and $\left.0.1^{\circ} \mathrm{C} / \mathrm{s}\right)$, which has been explained in detail in Supplementary information (Fig. S1). Another optimized parameter was the ligation buffer, which standardly contains DTT, known to reduce thiol bonds. Therefore, three DTT free buffers with different ionic contents were evaluated for their performance during the LCR (Table S2).

The final ligation reaction mixture used for the FO-SPR LCR calibration curves and sample analysis consisted of $10 \mu \mathrm{L}$ ligase buffer $\left(10 \times\right.$ concentrated), $10 \mu \mathrm{L}$ of $9^{\circ} \mathrm{N}$ enzyme $(10 \mathrm{u} / \mu \mathrm{L}), 2.5 \mu \mathrm{L}$ FW ligation probes ( $10 \mu \mathrm{M}$, for each target sequence), $2.5 \mu \mathrm{L}$ Rev ligation probes $(1 \mu \mathrm{M}$, for each target sequence), $50 \mu \mathrm{L}$ Au NPs $\left(0.5 \mathrm{nmol} / \mathrm{L}\right.$ in distilled nuclease free $\mathrm{H}_{2} \mathrm{O}$ ), $10 \mu \mathrm{L}$ target DNA, $15 \mu \mathrm{L}$ distilled nuclease free $\mathrm{H}_{2} \mathrm{O}$ making a total reaction volume of $100 \mu \mathrm{L}$. After adding all these components, the LCR mixture was mixed very carefully and covered with $60 \mu \mathrm{L}$ mineral oil (Immobiline DryStrip Cover Fluid, GE healthcare, Diegem, Belgium) to prevent evaporation during thermocycling.

\subsection{Data processing}

Data acquisition on the two spectrometers and NiDaq coupled thermocouples was done with the in-house developed LabView program (National Instruments, Austin, Texas). Once the SPR data and thermocouple data were combined, a first order derivative was calculated for each LCR melting cycle. The resulting melting peak was fitted using a Gaussian fit in Matlab (the mathworks, Natick, USA) to determine the melting temperature $\left(T_{\mathrm{m}}\right)$ and evaluate the melting peak quality for each LCR cycle.

\section{Results}

\subsection{LCR buffer optimization}

The first step in the implementation of the LCR on the FO-SPR platform was selection of the buffer compatible with both the FOSPR melting assay and the LCR. The standard ligation buffer supplied by the manufacturer of the ligation enzyme $9^{\circ} \mathrm{N}$ was not compatible with the FO-SPR assay due to the presence of DTT, which reduces thiol bonds essential for immobilization of DNA molecules on gold surfaces. Furthermore, an ionic strength of the buffer required optimization. The ionic concentration appears to be crucial for FO-SPR bioassays as it influences stability of the $\mathrm{Au}$ NPs, enzyme performance and hybridization speed and can thereby considerably affect assay sensitivity (Springer et al., 2010). Therefore, three different compositions of the standard ligation buffer (Table S2) were evaluated for their performance both in the LCR (Fig. 2) and the FO-SPR melting assay (Fig. 3).

The ligation yield was measured using qPCR High Resolution Melting (HRM) analysis on a ligated product. Here, the size of the melting signal was indicative for the ligation reaction yield. The results suggested that buffer 1 gave a ligation yield comparable to the standard buffer, confirming that DTT is not a necessary component for the LCR enzyme. On the other hand, doubling the amount of $\mathrm{MgCl}_{2}$ in buffer 2 drastically lowered the ligation yield, while the yield of LCR in buffer 3 was only a fraction lower than in the standard buffer. Because buffer 3 contains the same components as standard buffer, except for $60 \mathrm{mM} \mathrm{NaCl}$, these results clearly suggested that monovalent salts have a less drastic effect on the ligation yield in comparison with the divalent $\mathrm{MgCl}_{2}$. Based on these results, buffer 2 was excluded from further tests.

Next, buffers 1 and 3 were compared to evaluate the performance of the FO-SPR melting assay in these buffers. Fig. 3 


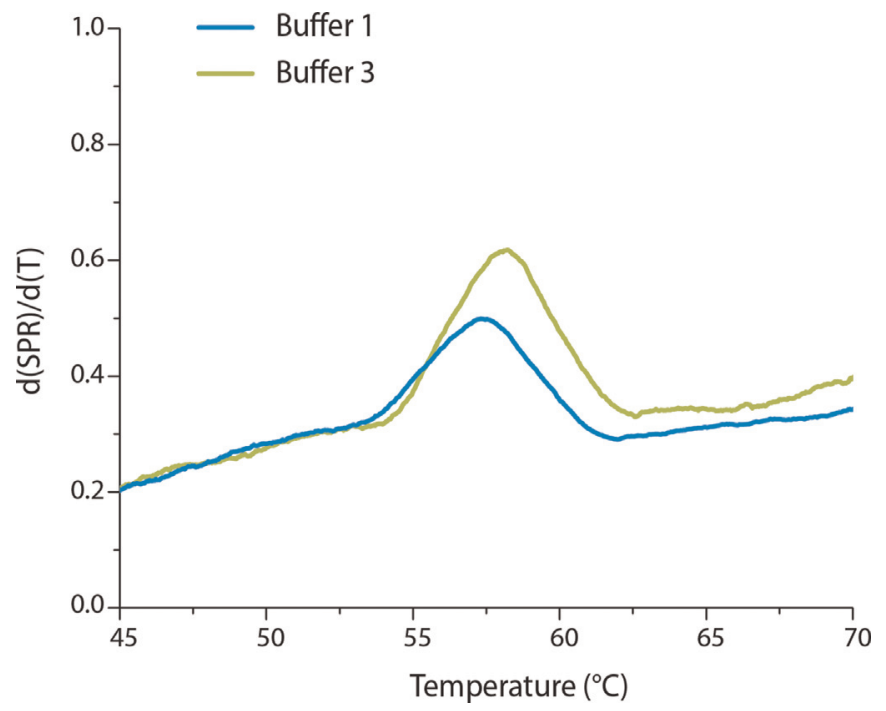

Fig. 3. FO-SPR melting analysis of Au NPs in two different buffers. Buffer 3 proved to be more suited than buffer 1 for the FO-SPR melting assay, as it resulted in the highest melting signal.

summarizes results of the FO-SPR melting assay as previously described by our group (Knez et al., 2012, 2013) for the $30 \mathrm{bp}$ target sequence in the two selected buffers. Here, buffer 3 proved to be more suited for the FO-SPR melting assay, as it resulted in the highest melting signal. These results were in line with obtained highest Au NP stability in this buffer (Supplementary information, Fig. S2).

\subsection{FO-SPR LCR assay}

As described in Fig. 1, the FO-SPR LCR consists of multiple ligation cycles, which are enabled by heating up and cooling down the reaction mixture. Because the heating of a fluid expands its volume, it also decreases the refractive index of the LCR mixture, resulting in the SPR signal that is the exact inverse of the temperature signal (Fig. 4, left graph). This temperature effect is

A - SPR channel 1

- SPR channel 2

---- Temperature

Cycle number

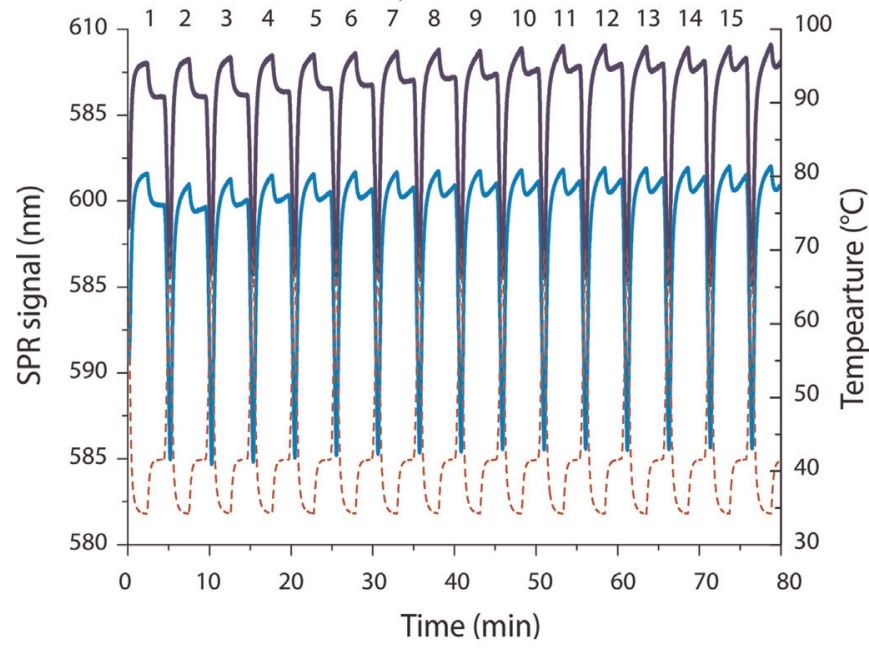

clearly visible in the FO-SPR signals on both measurement channels (left graph, SPR channel 1 and 2) and allows easy processing of the data, as the refractive index changes can be used to determine the reaction cycle boundaries. Each reaction cycle is split in 3 phases, namely the hybridization phase where the DNA target strands can hybridize with the ligation probes, the ligation phase where the ligation enzyme covalently links perfectly hybridized probes, and finally the melting phase where ligated products are melted from the target liberating it for the next round of ligation (Fig. 4, right graph).

The essential part of the LCR DNA amplification, for achieving the exponential amplification and thus a high detection limit $\left(10^{-9} \mathrm{M}\right.$ of target DNA), is the use of reverse ligation probe set. During the hybridization step of each LCR cycle, the forward ligation products can hybridize to detection probes on the FOSPR surface and Au NPs, forming a complex that is melted in the melting phase of each reaction cycle. Therefore, the acquired melting signal in the melting phase is uniquely attributable to the acquired LCR products and can thus be used to both identify and quantify the LCR product (Fig. S3). Importantly, Au NP and FOSPR probes cannot be ligated because they do not have a phosphate group necessary for the formation of a phosphodiester bond between the 3' hydroxyl group of Ligation probe FW (or Rev) 2 and the 5' phosphate group of Ligation probe FW (or Rev) 1.

\subsection{Quantification of DNA targets using FO-SPR LCR assay}

Next, the FO-SPR LCR assay was used to determine the target concentration by examining the number of LCR cycles necessary to obtain a FO-SPR melting point of the corresponding DNA target. The concept was first established using $100 \mathrm{fM}$ of a WT DNA target (Supplementary information, Fig. S4) and then subsequently implemented on 7 different target concentrations (100 nM, $10 \mathrm{nM}$, $1 \mathrm{nM}, 100 \mathrm{pM}, 10 \mathrm{pM}, 1 \mathrm{pM}$ and $100 \mathrm{fM}$ ) including a negative control without DNA target (no template control, NTC). For each concentration, the height of melting peaks were derived from all 40 LCR cycles and normalized to 1 . These values, represented in Fig. 5 (left graph) as a function of the total number of reaction cycles, pointed out that an increasing number of LCR cycles is necessary for lower target concentrations. At approximately the
B

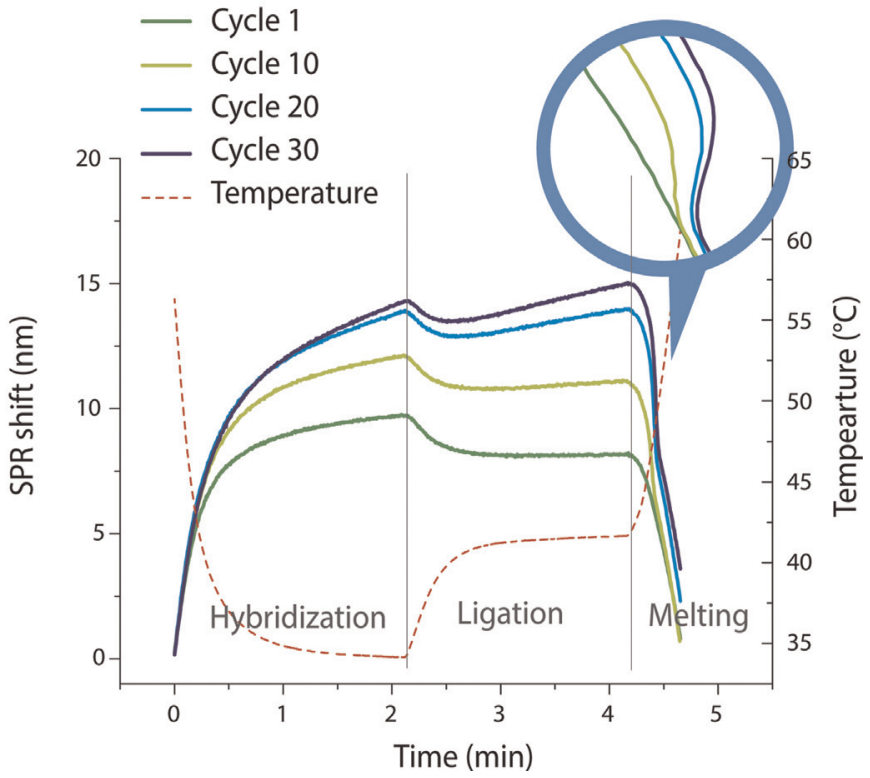

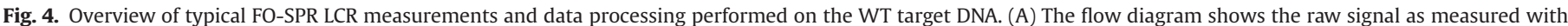
the FO-SPR device. (B) The obtained signal is further cut in individual reaction cycles. 


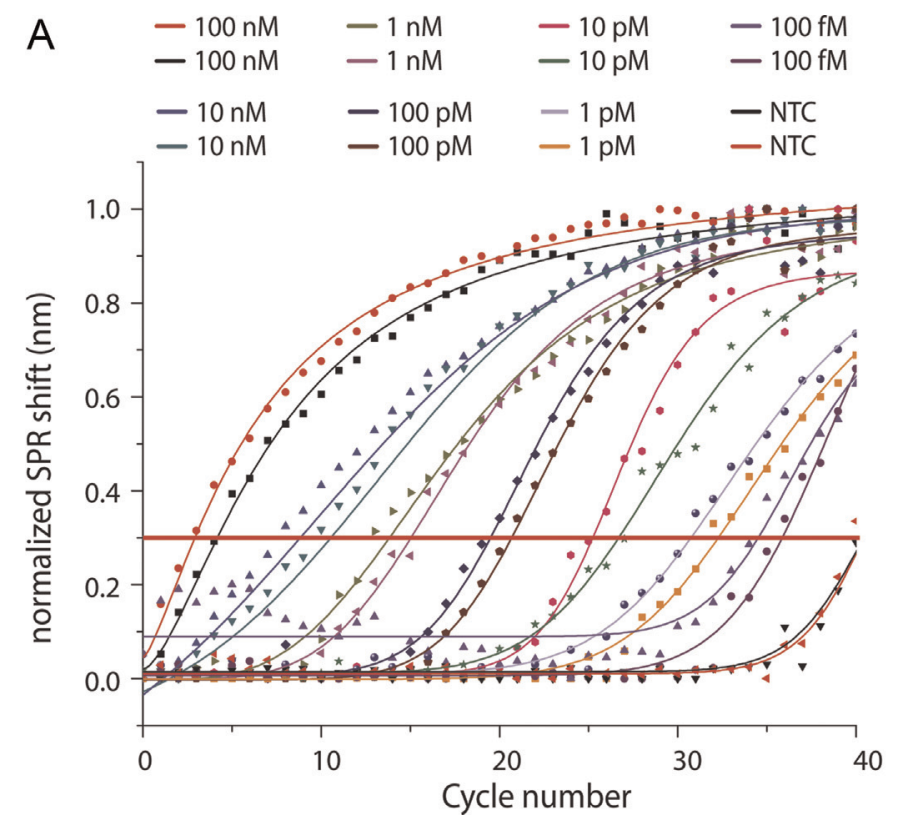

B

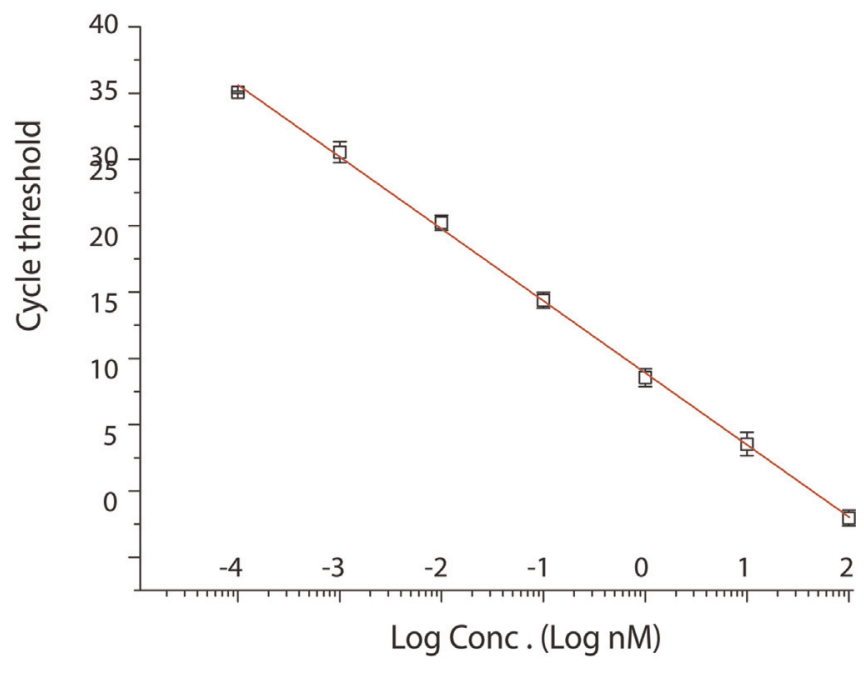

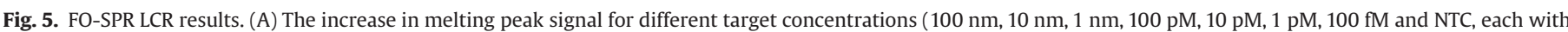

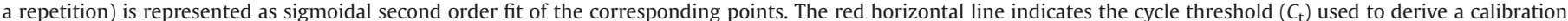

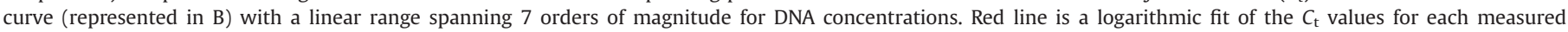

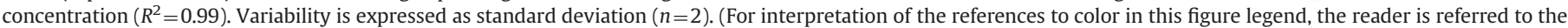
web version of this article.)

35th cycle, a positive melting signal became evident also for the NTC, suggesting either contamination of the reaction mixture with target DNA or the occurrence of non-specific amplification in the LCR assay at these later cycles. Next, the cycle threshold - being the minimal SPR shift above baseline - was chosen that is indicative of successful LCR amplification. By using a cycle threshold of 0.3 (horizontal red line in Fig. 5, left), $C_{t}$ values for each target concentration were derived and plotted as a function of the concentration, resulting in a calibration curve for the FO-SPR LCR assay (Fig. 5, right). The assay had a linear range spanning 7 orders of magnitude, which is only 1 order less than a conventional qPCR assay. The lowest concentration measured experimentally was $100 \mathrm{fM}$, as at lower concentrations the $\mathrm{Ct}$ was indistinguishable from the NTC, whereas the calculated LOD was $13.75 \mathrm{fM}$. Thus, the LCR improved the detection limit with 4 orders of magnitude compared to our previously published results on the FO-SPR

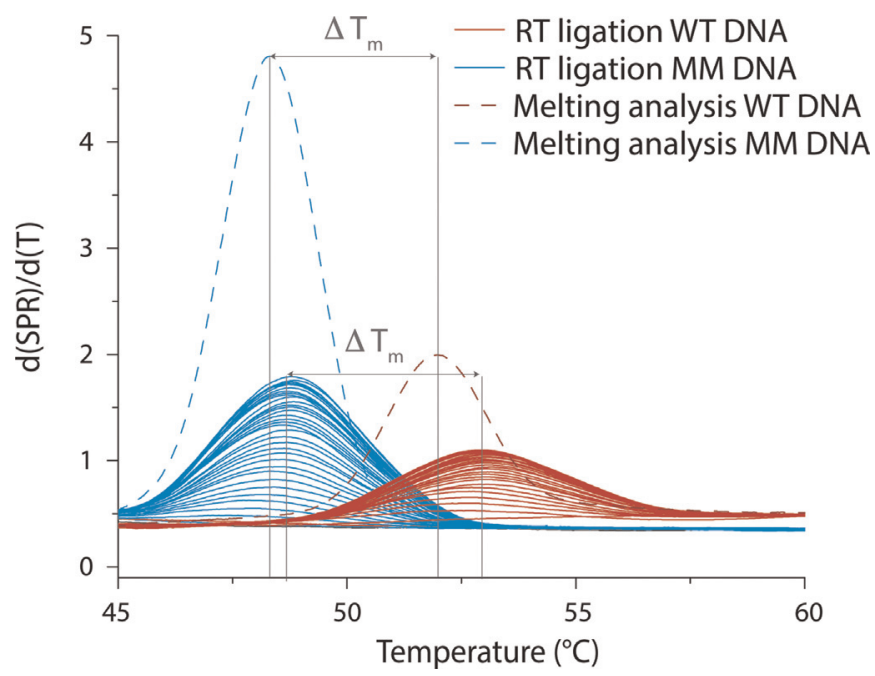

Fig. 6. Obtained signals for the FO-SPR LCR assay using WT and MM target DNA. The mutant clearly has a higher yield but a lower melting point (target $[C]=10 \mathrm{nM}$ ). melting assay (Knez et al., 2012, 2013). Although these results are still not matching the detection limit of qPCR (Meyer et al., 2008), a considerable improvement is possible. For instance, the ligation was not performed at the optimal ligation temperature of the enzyme $\left(45-90^{\circ} \mathrm{C}\right)$ because of the low hybridization temperature $\left(35^{\circ} \mathrm{C}\right)$, resulting in less than optimal assay (Barany, 1991). Better probe design and selection of targets would allow the LCR assay to be performed at higher hybridization temperatures, which can improve ligase performance and eliminate non-specific binding, thus further decreasing the detection limit.

\subsection{Target identification using FO-SPR LCR assay}

A final experiment was done to evaluate the performance of the FO-SPR LCR for SNP detection. In order to detect a SNP within the target DNA strand using LCR assay, two new ligation probes (Ligation probe FW 1 Mismatch and Ligation probe Rev 2 Mismatch, Table S1), matching the SNP in the target, needed to be introduced in the assay. This adjustment was necessary to fulfill the requirement of the ligation enzyme for perfect sequence complementarity between target strand and two ligation probes. The FO-SPR assay allows to discriminate the different LCR products because even a single base pair mismatch between the sensor immobilized hybridization probes and the ligated probes will shift the $T_{\mathrm{m}}$ to lower temperatures. Furthermore, mutations in the target sequence that will not affect the ligation reaction can also be discriminated with the FO-SPR assay. This means that not all mutations in the target sequence have to be known.

The two LCRs performed both for WT and the MM target were compared with a melting analysis of the same targets performed as described previously in our work (Knez et al., 2012). Although the MM target clearly had a lower $T_{\mathrm{m}}$ value compared to the WT, as expected, it surprisingly showed better yield in the LCR (Fig. 6). This can be explained by a difference in hybridization yield. The MM ligation probes are hybridized at a temperature further below the melting point of probes and targets, which is known to give a good hybridization yield (Rychlik et al., 1990). As for the WT ligation probes, the hybridization temperature is less optimal, 
explaining why there is a difference in ligation yield. Importantly, the $T_{\mathrm{m}}$ measured during the LCR allowed discrimination of the SNP bearing target from the WT target. In comparison with the melting analysis, which is performed at a lower ramping speed, the melting signal obtained from the LCR assay was considerably lower with wider melting peaks. However, the resolution of the fast melting cycles was better with a $\Delta T_{\mathrm{m}}$ between WT and MM target of $4.3^{\circ} \mathrm{C}$ compared to $3.8^{\circ} \mathrm{C}$ in the slow melting analysis. Similar trends in $T_{\mathrm{m}}$ were also visible during the optimization experiments for thermal ramping speed. It can be speculated that the increased ramping speed results in the formation of less bonds between the Au NPs and the FO-SPR surface through the target DNA, which broadens the melting transition (Jin et al., 2003).

\section{Conclusions}

Integration of LCR with the FO-SPR melting assay considerably increased the value of both assays. The combined assay allowed quantifying and identifying DNA targets over a wide concentration range. Moreover, the assay could discriminate SNPs without any post reaction analysis. The integration of the two assays required initial optimization, especially buffer ionic strength and thermal ramping speed as these parameters both influence the ligation enzyme performance and the hybridization yield of the Au NPs on the FO-SPR sensor. Here, the higher temperature ramping speeds were selected for at the cost of melting resolution as the assay speed is an important factor for its future applications. Nonetheless, even at a ramping speed 10 times higher than classic FO-SPR DNA melting assays, the discrimination of SNP bearing targets was still feasible with high precision. The FO-SPR LCR assay had a LOD of $13.75 \mathrm{fM}$. Better ligation probe design and selection of optimal ligation conditions could decrease the LOD even further to a similar level as PCR. Finally, in comparison with earlier described LCR assays, the FO-SPR implementation offered the advantage of cycle-to-cycle information on the amplified target strands, eliminating any time-consuming post reaction analysis steps. Therefore, the FO-SPR LCR assay represents a great improvement in development of fast POC tests.

\section{Acknowledgements}

The research leading to these results has received funding from the European Commission's Seventh Framework Programme (FP7/ 2007-2013) under the grant agreement BIOMAX (project no. 264737), from the KU Leuven Industrial Research Fund (IOF/KP/ 12/002 NanoDiag) and the Flemish Institute for the Promotion of Innovation through Science and Development (IWT 100936
Sensors for Food VIS-traject, IWT/110770 LA-traject and IWT/ 120584/Innovatiemandaat/Optische vezel SPR biosensoren of F.D.).

\section{Appendix A. Supplementary information}

Supplementary data associated with this article can be found in the online version at http://dx.doi.org/10.1016/j.bios.2014.08.067.

\section{References}

Barany, F., 1991. PCR Methods Appl. 1, 5-16.

Cao, Y.C., Jin, R., Thaxton, C.S., Mirkin, C.A., 2005. Talanta 67, 449-455.

Cheng, J.C., Huang, C.L., Lin, C.C., Chen, C.C., Chang, Y.C., Chang, S.S., Tseng, C.P., 2006 Clin. Chem. 52, 1997-2004.

Delport, F., Pollet, J., Janssen, K., Verbruggen, B., Knez, K., Spasic, D., Lammertyn, J., 2012. Nanotechnology 23 (6), 065503.

Espy, M.J., Uhl, J.R., Sloan, L.M., Buckwalter, S.P., Jones, M.F., Vetter, E.A., Yao, J.D. Wengenack, N.L., Rosenblatt, J.E., Cockerill 3rd, F.R., Smith, T.F., 2006. Clin Microbiol. Rev. 19 (3), 595.

Fiche, J.B., Fuchs, J., Buhot, A., Calemczuk, R., Livache, T., 2008. Anal. Chem. 80 1049-1057.

Gervais, L., de Rooij, N., Delamarche, E., 2011. Adv. Mater. 23, H151-H176.

He, L., Musick, M.D., Nicewarner, S.R., Salinas, F.G., Benkovic, S.J., Natan, M.J., Keating C.D. 2000. Am. Chem. Soc. 122 (38), 9071-9077.

Hurst, S.J., Lytton-Jean, A.K., Mirkin, C.A., 2006. Anal. Chem. 78, 8313-8318.

Jans, H., Huo, Q., 2012. Chem. Soc. Rev. 41, 2849-2866.

Janssen, K.P., Knez, K., Vanysacker, L., Schrooten, J., Spasic, D., Lammertyn, J., 2012 Nanotechnology 23, 235503.

Jin, R.C., Wu, G.S., Li, Z., Mirkin, C.A., Schatz, G.C., 2003. J. Am. Chem. Soc. 125 1643-1654.

Knez, K., Janssen, K.P., Pollet, J., Spasic, D., Lammertyn, J., 2012. Small 8 (6), 868-872.

Knez, K., Janssen, K.P., Spasic, D., Declerck, P., Vanysacker, L., Denis, C., Tran, D.T., Lammertyn, J., 2013. Anal. Chem. 85 (3), 1734-1742.

Lee, C.Y., Gong, P., Harbers, G.M., Grainger, D.W., Castner, D.G., Gamble, L.J., 2006. Anal. Chem. 78, 3316-3325.

Lytton-Jean, A.K.R., Mirkin, C.A., 2005. J. Am. Chem. Soc. 127, 12754-12755.

Meyer, M., Briggs, A.W., Maricic, T., Höber, B., Höffner, B., Krause, J., Weihmann, A Pääbo, S., Hofreiter, M., 2008. Nucleic Acids Res. 36 (1), e5.

Moon, S., Kim, D.J., Kim, K., Kim, D., Lee, H., Lee, K., Haam, S., 2010. Appl. Opt. 49, 484-491.

Oh, J.H., Lee, J.S., 2011. Anal. Chem. 83, 7364-7370.

Pingel, J., Buhot, A., Calemczuk, R., Livache, T., 2012. Biosens. Bioelectron. 31, 554-557.

Pollet, J., Delport, F., Janssen, K.P., Jans, K., Maes, G., Pfeiffer, H., Wevers, M., Lammertyn, J., 2009. Biosens. Bioelectron. 25 (4), 864-869.

Pollet, J., Delport, F., Janssen, K., Tran, T., Wouters, J., Verbiest, T., Lammertyn, J., 2011. Talanta 83 (5), 1436-1441.

Rychlik, W., Spencer, W.J., Rhoads, R.E., 1990. Nucleic Acids Res. 18, 6409-6412.

Sando, S., Abe, H., Kool, E.T., 2004. J. Am. Chem. Soc. 126, 1081-1087.

Shen, W., Deng, H., Gao, Z., 2012. J. Am. Chem. Soc. 134, 14678-14681.

Storhoff, J.J., Lucas, A.D., Garimella, V., Bao, Y.P., Muller, U.R., 2004. Nat. Biotechnol. 22, 883-887.

Springer, T., Sipova, H., Vaisocherova, H., Stepanek, J., Homola, J., 2010. Nucleic Acids Res. 38, 7343-7351.

Tran, T., Knez, K., Janssen, K., Pollet, J., Spasic, D., Lammertyn, J., 2013. Biosens. Bioelectron. 43, 245-251 